\title{
NASH EQUILIBRIUM FOR BINARY CONVEXITIES
}

\author{
TARAS RADUL
}

\begin{abstract}
This paper is devoted to Nash equilibrium for games in capacities. Such games with payoff expressed by the Choquet integral were considered by Kozhan and Zarichnyi (2008) and existence of Nash equilibrium was proved. We also consider games in capacities but with expected payoff expressed by the Sugeno integral. We prove existence of Nash equilibrium in a general context of abstract binary (non-linear) convexity and then we obtain an existence theorem for games in capacities.
\end{abstract}

\section{Introduction}

The classical Nash equilibrium theory is based on fixed point theory and was developed in the frame of linear convexity with mixed strategies of a player being probability (additive) measures on a set of pure strategies. In last decades the interest in Nash equilibria in more general frames is rapidly growing. For instance, Briec and Horvath proved in [1] existence of a Nash equilibrium point for $B$-convexity and MaxPlus convexity which are non-linear. Let us remark that MaxPlus convexity is related to idempotent (Maslov) measures in the same sense as linear convexity is related to probability measures.

We can use additive measures only when we know precisely probabilities of all events considered in a game. However, this is not the case in many modern economic models. The decision theory under uncertainty considers a model when probabilities of states are either not known or imprecisely specified. Gilboa [5]

2010 Mathematics Subject Classification. 18C15, 52A01, 54B30, 54H25, 91A10

Key words and phrases. Nash equilibrium; game in capacities; Sugeno integral; binary convexity. 
and Schmeidler [17] axiomatized expectations expressed by Choquet integrals attached to non-additive measures called capacities, as a formal approach to decision-making under uncertainty.

An alternative to the so-called Choquet expected utility model is the qualitative decision theory. The corresponding expected utility is expressed by the Sugeno integral. See the papers [3], [4], [2], [16] and others for more details about the qualitative decision theory and motivation of using the Sugeno integral.

Kozhan and Zarichnyi introduced in [7] a notion of Nash equilibrium of a game where players are allowed to form non-additive beliefs about opponent's decision but also to play their mixed non-additive strategies. Such game was called by the authors as the game in capacities. The expected payoff function was defined using the Choquet integral. Kozhan and Zarichnyi proved an existence theorem using a linear convexity on the space of capacities which is preserved by the Choquet integral. The problem of existence of Nash equilibrium for other functors was stated in [7].

In this paper, following [7], we introduce a concept of Nash equilibrium for a game in capacities. However, motivated by the qualitative approach, we consider an expected payoff function defined by the Sugeno integral. In order to prove an existence theorem for this particular case, we consider a more general framework which could unify all situations mentioned before and give us a method to prove theorems about existence of Nash equilibrium in different contexts. We use categorical methods and abstract convexity theory.

The notion of convexity considered in this paper is considerably broader than the classical one; in particular, it is not restricted to the context of linear spaces. Such convexities appeared in the process of studying different structures like partially ordered sets, semilattices, lattices, superextensions etc. We base our approach on the notion of topological convexity from [20] where the general convexity theory is covered from axioms to applications in different areas. Particularly, this book contains the Kakutani fixed point theorem for abstract convexity.

The above mentioned constructions of spaces of probability measures, idempotent measures and capacities are functorial and can be completed to monads (see [15], [22] and [11] for more details). A convexity structure on each $\mathbb{F}$-algebra for any monad $\mathbb{F}$ in the category of compact Hausdorff spaces and continuous maps was introduced in [12].

We prove a counterpart of the Nash theorem for an abstract convexity. Particularly, we consider binary convexities. These results are used to obtain a Nash type theorem for algebras of any $L$-monad with binary convexity. Since a capacity monad is an $L$-monad with binary convexity [13], we obtain as corollary the corresponding result for capacities. 


\section{Binary convexities}

A family $\mathcal{C}$ of closed subsets of a compactum $X$ is called a convexity on $X$ if $\mathcal{C}$ is stable for intersection, and contains $X$ and the empty set. The elements of $\mathcal{C}$ are called $\mathcal{C}$-convex (or simply convex). Although we follow the general concept of abstract convexity from [20], our definition is different. We consider only closed convex sets. Such structure is called a closure structure in [20]. Our definition is the same as in [21]. The whole family of convex sets in the sense of [20] could be obtained by the operation of union of up-directed families. In what follows, we assume that each convexity contains all singletons.

A convexity $\mathcal{C}$ on $X$ is called $T_{2}$ if for any distinct $x_{1}, x_{2} \in X$ there exist $S_{1}, S_{2} \in \mathcal{C}$ such that $S_{1} \cup S_{2}=X, x_{1} \notin S_{2}$ and $x_{2} \notin S_{1}$. Let us remark that if a convexity $\mathcal{C}$ on a compactum $X$ is $T_{2}$, then $\mathcal{C}$ is a subbase for closed sets. A convexity $\mathcal{C}$ on $X$ is called $T_{4}$ (normal) if for any disjoint $C_{1}, C_{2} \in \mathcal{C}$ there exist $S_{1}, S_{2} \in \mathcal{C}$ such that $S_{1} \cup S_{2}=X, C_{1} \cap S_{2}=\emptyset$ and $C_{2} \cap S_{1}=\emptyset$.

Let $(X, \mathcal{C}),(Y, \mathcal{D})$ be two compacta with convexity structures. A continuous map $f: X \rightarrow Y$ is called a $C P$ map (convexity preserving map) if $f^{-1}(D) \in \mathcal{C}$ for each $D \in \mathcal{D} ; f$ is called a $C C$ map (convex-to-convex map) if $f(C) \in \mathcal{D}$ for each $C \in \mathcal{C}$.

By a multimap (set-valued map) of a set $X$ into a set $Y$ we mean a map $F: X \rightarrow 2^{Y}$ and we use the notation $F: X \multimap Y$. If $X$ and $Y$ are topological spaces, then a multimap $F: X \multimap Y$ is called upper semi-continuous (USC) provided for each open set $O \subset Y$ the set $\{x \in X \mid F(x) \subset O\}$ is open in $X$. It is well-known that a multimap with compact values is USC iff its graph is closed in $X \times Y$.

Let $F: X \multimap X$ be a multimap. We say that a point $x \in X$ is a fixed point of $F$ if $x \in F(x)$. The following counterpart of the Kakutani theorem for an abstract convexity is a partial case of Theorem 3 from [21] (it also could be obtained combining Theorem 6.15, Chapter IV and Theorem 4.10, Chapter III from $[20])$.

TheOREM 2.1. Let $\mathcal{C}$ be a normal convexity on a compactum $X$ such that all convex sets are connected and $F: X \multimap X$ is a USC multimap with values in $\mathcal{C}$. Then $F$ has a fixed point.

Let $\mathcal{C}$ be a family of subsets of a compactum $X$. We say that $\mathcal{C}$ is linked if the intersection of any two its elements is non-empty. A convexity $\mathcal{C}$ is called binary if the intersection of every its linked subsystem of $\mathcal{C}$ is non-empty.

Lemma 2.2. Let $\mathcal{C}$ be a $T_{2}$ binary convexity on a continuum $X$. Then $\mathcal{C}$ is normal and all convex sets are connected.

Proof. The first assertion of the lemma is proved in [15, Lemma 3.1]. Let us prove the second one. Consider any $A \in \mathcal{C}$. A retraction $h_{A}: X \rightarrow A$ is 
defined in [9] by the formula $h_{A}(x)=\cap\{C \in \mathcal{C} \mid x \in C$ and $C \cap A \neq \emptyset\}$. Hence $A$ is connected.

Now we can reformulate Theorem 2.1 for binary convexities.

Theorem 2.3. Let $\mathcal{C}$ be a $T_{2}$ binary convexity on a continuum $X$ and let $F: X \multimap X$ be a USC multimap with values in $\mathcal{C}$. Then $F$ has a fixed point.

Let us recall the definition of Nash equilibrium. We consider an $n$-players game $f: X=\prod_{i=1}^{n} X_{i} \rightarrow \mathbb{R}^{n}$ with compact Hausdorff spaces of strategies $X_{i}$. The coordinate function $f_{i}: X \rightarrow \mathbb{R}$ is called the payoff function of $i$-th player. For $x \in X$ and $t_{i} \in X_{i}$ we use the notation $\left(x ; t_{i}\right)=\left(x_{1}, \ldots, x_{i-1}, t_{i}, x_{i+1}, \ldots, x_{n}\right)$. A point $x \in X$ is called a Nash equilibrium point if for each $i \in\{1, \ldots, n\}$ and for each $t_{i} \in X_{i}$ we have $f_{i}\left(x ; t_{i}\right) \leq f_{i}(x)$.

Now, let $\mathcal{C}_{i}$ be a convexity on $X_{i}$. We say that a function $f_{i}: X \rightarrow \mathbb{R}$ is quasiconcave with respect to the $i$-th variable if we have $\left(f_{i}^{x}\right)^{-1}([t ;+\infty)) \in \mathcal{C}_{i}$ for all $t \in \mathbb{R}$ and $x \in X$ where $f_{i}^{x}: X_{i} \rightarrow \mathbb{R}$ is the function defined as follows: $f_{i}^{x}\left(t_{i}\right)=f_{i}\left(x ; t_{i}\right)$ for $t_{i} \in X_{i}$.

THEOREM 2.4. Let $f: X=\prod_{i=1}^{n} X_{i} \rightarrow \mathbb{R}^{n}$ be a game with a normal convexity $\mathcal{C}_{i}$ defined on each compactum $X_{i}$ such that all convex sets are connected, the function $f$ is continuous and the function $f_{i}: X \rightarrow \mathbb{R}$ is quasiconcave with respect to the $i$-th variable for each $i \in\{1, \ldots, n\}$. Then there exists a Nash equilibrium point.

Proof. Fix any $x \in X$. For each $i \in\{1, \ldots, n\}$ let

$$
M_{i}^{x}=\left\{t \in X_{i} \mid f_{i}^{x}(t)=\max _{s \in X_{i}} f_{i}^{x}(s)\right\} .
$$

Then $M_{i}^{x}$ is a closed subset $X_{i}$. Since the function $f_{i}: X \rightarrow \mathbb{R}$ is quasiconcave with respect to the $i$-th variable, $M_{i}^{x} \in \mathcal{C}_{i}$. Define a multimap $F: X \multimap X$ by the formula $F(x)=\prod_{i=1}^{n} M_{i}^{x}$ for $x \in X$.

Let us show that $F$ is USC. Consider any point $(x, y) \in X \times X$ such that $y \notin F(x)$. Then there exists $i \in\{1, \ldots, n\}$ such that $f_{i}^{x}\left(y_{i}\right)<\max _{s \in X_{i}}\left\{f_{i}^{x}(s)\right\}$. Hence we can choose $t_{i} \in X_{i}$ such that $f_{i}\left(x ; y_{i}\right)<f_{i}\left(x ; t_{i}\right)$. Since $f_{i}$ is continuous, there exist a neighbourhood $O_{x}$ of $x$ in $X$ and a neighbourhood $O_{y_{i}}$ of $y_{i}$ in $Y_{i}$ such that for any $x^{\prime} \in O_{x}$ and $y_{i}^{\prime} \in O_{y_{i}}$ we have $f_{i}\left(x ; y_{i}^{\prime}\right)<f_{i}\left(x ; t_{i}\right)$. Put $O_{y}=\left(\operatorname{pr}_{i}\right)^{-1}\left(O_{y_{i}}\right)$. Then for each $\left(x^{\prime}, y^{\prime}\right) \in O_{x} \times O_{y}$ we have $y^{\prime} \notin F\left(x^{\prime}\right)$. Thus the graph of $F$ is closed in $X \times Y$, hence $F$ is upper semicontinuous.

We consider on $X$ the family $\mathcal{C}=\left\{\prod_{i=1}^{n} C_{i} \mid C_{i} \in \mathcal{C}_{i}\right\}$. It is easy to see that $\mathcal{C}$ forms a normal convexity on the compactum $X$ such that all convex sets are connected. Then, by Theorem 2.1, $F$ has a fixed point which is a Nash equilibrium point. 
Now, the previous theorem and Lemma 2.2 imply the following corollary.

Corollary 2.5. Let $f: X=\prod_{i=1}^{n} X_{i} \rightarrow \mathbb{R}^{n}$ be a game such that a $T_{2}$ binary convexity $\mathcal{C}_{i}$ is defined on each continuum $X_{i}$, the function $f$ is continuous and the function $f_{i}: X \rightarrow \mathbb{R}$ is quasiconcave with respect to the $i$-th variable for each $i \in\{1, \ldots, n\}$. Then there exists a Nash equilibrium point.

\section{3. $L$-monads and its algebras}

By Comp we denote the category of compact Hausdorff spaces (compacta) and continuous maps. For each compactum $X$ we denote by $C(X)$ the Banach space of all continuous functions on $X$ with the usual sup-norm. In what follows, all spaces and maps are assumed to be in Comp except for $\mathbb{R}$ and maps in sets $C(X)$ with $X$ compact Hausdorff.

We apply Corollary 2.5 to study games defined on the algebras of binary $L$-monads. We recall some categorical notions (see [8] and [19] for more details). We define them only for the category Comp. Let $F:$ Comp $\rightarrow$ Comp be a covariant functor. A functor $F$ is called continuous if it preserves the limits of inverse systems. In what follows, all functors are assumed to preserve monomorphisms, epimorphisms, weight of infinite compacta. We also assume that our functors are continuous. For a functor $F$ which preserves monomorphisms and for an embedding $i: A \rightarrow X$ we shall identify the space $F A$ and the subspace $F(i)(F A) \subset F X$.

A monad $\mathbb{T}=(T, \eta, \mu)$ in the category Comp consists of an endofunctor $T:$ Comp $\rightarrow$ Comp and natural transformations $\eta: \operatorname{Id}_{\text {Comp }} \rightarrow T$ (unity), $\mu: T^{2} \rightarrow$ $T$ (multiplication) satisfying the relations $\mu \circ T \eta=\mu \circ \eta T=\mathbf{1}_{T}$ and $\mu \circ \mu T=\mu \circ T \mu$. (By Id $\mathrm{I}_{\text {Comp }}$ we denote the identity functor on the category Comp and $T^{2}$ is the superposition $T \circ T$.)

Let $\mathbb{T}=(T, \eta, \mu)$ be a monad in the category Comp. A pair $(X, \xi)$, where $\xi: T X \rightarrow X$ is a map, is called a $\mathbb{T}$-algebra if $\xi \circ \eta X=\operatorname{id}_{X}$ and $\xi \circ \mu X=\xi \circ T \xi$. Let $(X, \xi),\left(Y, \xi^{\prime}\right)$ be two $\mathbb{T}$-algebras. A map $f: X \rightarrow Y$ is called a morphism of $\mathbb{T}$-algebras if $\xi^{\prime} \circ T f=f \circ \xi$.

Let $(X, \xi)$ be an $\mathbb{F}$-algebra for a monad $\mathbb{F}=(F, \eta, \mu)$ and let $A$ be a closed subset of $X$. Denote by $f_{A}$ the quotient map $f_{A}: X \rightarrow X / A$ (the equivalence classes are one-point sets $\{x\}$ for $x \in X \backslash A$ and the set $A$ ) and put $a=f_{A}(A)$. Denote $A^{+}=\left(F f_{A}\right)^{-1}(\eta(X / A)(a))$. Define the $\mathbb{F}$-convex hull $\operatorname{conv}_{\mathbb{F}}(A)$ of $A$ as follows $\operatorname{conv}_{\mathbb{F}}(A)=\xi\left(A^{+}\right)$. Put additionally $\operatorname{conv}_{\mathbb{F}}(\emptyset)=\emptyset$. We define the family $\mathcal{C}_{\mathbb{F}}(X, \xi)=\left\{A \subset X \mid A\right.$ is closed and $\left.\operatorname{conv}_{\mathbb{F}}(A)=A\right\}$. The elements of the family $\mathcal{C}_{\mathbb{F}}(X, \xi)$ will be called $\mathbb{F}$-convex. It was shown in [12] that the family $\mathcal{C}_{\mathbb{F}}(X, \xi)$ forms a convexity on $X$, moreover, each morphism of $\mathbb{F}$-algebras is a CP map. Let us remark that the one-point sets are always $\mathbb{F}$-convex. 
We do not know if the introduced convexities are $T_{2}$. In this section we consider a class of monads generating convexities which have this property. The class of $L$-monads was introduced in [12] and it contains many well-known monads in Comp like superextension, hyperspace, probability measure, capacity, idempotent measure, etc. For $\phi \in C(X)$ by $\max \phi$ (respectively, $\min \phi$ ) we denote $\max _{x \in X} \phi(x)$ (respectively, $\left.\min _{x \in X} \phi(x)\right)$ and $\pi_{\phi}$ or $\pi(\phi)$ denote the corresponding projection $\pi_{\phi}: \prod_{\psi \in C(X)}[\min \psi, \max \psi] \rightarrow[\min \phi, \max \phi]$. It was shown in [14] that for each $L$-monad $\mathbb{F}=(F, \eta, \mu)$ the space $F X$ can be considered as a subset of the product $\prod_{\phi \in C(X)}[\min \phi, \max \phi]$, moreover, we have $\pi_{\phi} \circ \eta X=\phi, \pi_{\phi} \circ \mu X=\pi\left(\pi_{\phi}\right)$ for all $\phi \in C(X)$ and $\pi_{\psi} \circ F f=\pi_{\psi \circ f}$ for all $\psi \in C(Y), f: X \rightarrow Y$. We can consider these properties of $L$-monads as a definition [14].

We say that an $L$-monad $\mathbb{F}=(F, \eta, \mu)$ weakly preserves preimages if for each map $f: X \rightarrow Y$ and each closed subset $A \subset Y$ we have

$$
\pi_{\phi}(\nu) \in\left[\min \phi\left(f^{-1}(A)\right), \max \phi\left(f^{-1}(A)\right)\right]
$$

for each $\nu \in(F f)^{-1}(A)$ and $\phi \in C(X)$ [12]. It was shown in [12] that for each $L$-monad $\mathbb{F}$ which weakly preserves preimages the convexity $\mathcal{C}_{\mathbb{F}}(F X, \mu X)$ is $T_{2}$.

LEMmA 3.1. Let $(X, \xi)$ be an $\mathbb{F}$-algebra for an L-monad $\mathbb{F}=(F, \eta, \mu)$ which weakly preserves preimages. Then the map $\xi: F X \rightarrow X$ is a CC map for convexities $\mathcal{C}_{\mathbb{F}}(F X, \mu)$ and $\mathcal{C}_{\mathbb{F}}(X, \xi)$, respectively.

Proof. Consider any $B \in \mathcal{C}_{\mathbb{F}}(F X, \mu)$. We should show that $\xi(B) \in \mathcal{C}_{\mathbb{F}}(X, \xi)$. Denote by $\chi: X \rightarrow X / \xi(B)$ the quotient map and put $b=\chi(\xi(B))$. Consider any $\mathcal{A} \in F X$ such that $F \chi(\mathcal{A})=\eta(X / \xi(B))(b)$. We should show that $\xi(\mathcal{A}) \in \xi(B)$.

Consider the quotient map $\chi_{1}: F X \rightarrow F X / B$ and put $b_{1}=\chi_{1}(B)$. There exists a (unique) continuous map $\xi^{\prime}: F X / B \rightarrow X / \xi(B)$ such that $\xi^{\prime}\left(b_{1}\right)=b$ and $\xi^{\prime} \circ \chi_{1}=\chi \circ \xi$. Put $\mathcal{D}=F(\eta X)(\mathcal{A})$. We have $F \xi(\mathcal{D})=\mathcal{A}$, hence $F \xi^{\prime} \circ F \chi_{1}(\mathcal{D})=$ $F \chi \circ F \xi(\mathcal{D})=F \chi(\mathcal{A})=\eta(X / \xi(B))(b)$. Since $F$ weakly preserves preimages, we have $F \chi_{1}(\mathcal{D})=\eta(F X / B)\left(b_{1}\right)$. Since $B \in \mathcal{C}_{\mathbb{F}}(F X, \mu)$, we have $\mu X(\mathcal{D}) \in B$. Hence, $\xi(\mathcal{A})=\xi \circ F \xi(\mathcal{D})=\xi \circ \mu(\mathcal{D}) \in \xi(B)$.

We call a monad $\mathbb{F}$ binary if $\mathcal{C}_{\mathbb{F}}(X, \xi)$ is binary for each $\mathbb{F}$-algebra $(X, \xi)$.

Lemma 3.2. Let $\mathbb{F}=(F, \eta, \mu)$ be a binary L-monad which weakly preserves preimages. Then for each $\mathbb{F}$-algebra $(X, \xi)$ the convexity $\mathcal{C}_{\mathbb{F}}(X, \xi)$ is $T_{2}$.

Proof. Consider any two distinct points $x, y \in X$. Being a morphism of $\mathbb{F}$ algebras $(F X, \mu X)$ and $(X, \xi)$ the map $\xi$ is a CP map and we have $\xi^{-1}(x), \xi^{-1}(y)$ $\in \mathcal{C}_{\mathbb{F}}(F X, \mu)$. Since $\mathcal{C}_{\mathbb{F}}(F X, \mu)$ is $T_{2}$ and binary, it is normal, by Lemma 2.2. Hence we can choose $L_{1}, L_{2} \in \mathcal{C}_{\mathbb{F}}(F X, \mu)$ such that $L_{1} \cup L_{2}=F X$ and $L_{1} \cap$ $\xi^{-1}(x)=\emptyset, L_{2} \cap \xi^{-1}(y)=\emptyset$. Then we have $\xi\left(L_{1}\right), \xi\left(L_{2}\right) \in \mathcal{C}_{\mathbb{F}}(X, \xi)$, by Lemma 3.1, $\xi\left(L_{1}\right) \cup \xi\left(L_{2}\right)=X, x \notin L_{1}$ and $y \notin L_{2}$. 
Consider any $L$-monad $\mathbb{F}=(F, \eta, \mu)$. It is easy to check that for each segment $[a, b] \subset \mathbb{R}$ the pair $\left([a, b], \xi_{[a, b]}\right)$ is an $\mathbb{F}$-algebra where $\xi_{[a, b]}=\pi_{\mathrm{id}_{[a, b]}}$. Consider a game $f: X=\prod_{i=1}^{n} X_{i} \rightarrow \mathbb{R}^{n}$ where for each compactum $X_{i}$ there exists a map $\xi_{i}: F X_{i} \rightarrow X_{i}$ such that the pair $\left(X_{i}, \xi_{i}\right)$ is an $\mathbb{F}$-algebra. We say that the function $f_{i}: X \rightarrow \mathbb{R}$ is a morphism of $\mathbb{F}$-algebras with respect to the $i$-th variable if for each $x \in X$ the function $f_{i}^{x}: X_{i} \rightarrow \mathbb{R}$ is a morphism of $\mathbb{F}$-algebras $\left(X_{i}, \xi_{i}\right)$ and $\left(\left[\min f_{i}^{x}, \max f_{i}^{x}\right], \xi_{\left[\min f_{i}^{x}, \max f_{i}^{x}\right]}\right)$.

Theorem 3.3. Let $\mathbb{F}=(F, \eta, \mu)$ be a binary $L$-monad which weakly preserves preimages. Let $f: X=\prod_{i=1}^{n} X_{i} \rightarrow \mathbb{R}^{n}$ be a game such that an $\mathbb{F}$-algebra map $\xi_{i}: F X_{i} \rightarrow X_{i}$ is defined on each continuum $X_{i}$, the function $f$ is continuous and the function $f_{i}: X \rightarrow \mathbb{R}$ is a morphism of $\mathbb{F}$-algebras with respect to the $i$-th variable for each $i \in\{1, \ldots, n\}$. Then there exists a Nash equilibrium point.

Proof. Since for each $x \in X$ the function $f_{i}^{x}: X_{i} \rightarrow \mathbb{R}$ is a morphism of $\mathbb{F}$-algebras, it is a CP map, hence quasiconcave. Now, our theorem follows from Lemma 3.2 and Corollary 2.5.

\section{Pure and mixed strategies}

Let $\mathbb{F}=(F, \eta, \mu)$ be a binary $L$-monad which weakly preserves preimages. In this section we consider Nash equilibrium for free algebras $(F X, \mu X)$. The points of a compactum $X$ are called pure strategies and the points of $F X$ are called mixed strategies. Such approach is a natural generalization of the model from [7] where spaces of capacities were considered.

We consider a game $u: X=\prod_{i=1}^{n} X_{i} \rightarrow \mathbb{R}^{n}$ with compact Hausdorff spaces of pure strategies $X_{1}, \ldots, X_{n}$ and continuous payoff functions $u_{i}: \prod_{i=1}^{n} X_{i} \rightarrow \mathbb{R}$.

It is well-known how to construct the tensor product of two (or finite number) probability measures. This operation was generalized in [19] over any monad in the category Comp. More precisely, for any compacta $X_{1}, \ldots, X_{n}$ a continuous map $\otimes: \prod_{i=1}^{n} F X_{i} \rightarrow F\left(\prod_{i=1}^{n} X_{i}\right)$ was constructed such that $\otimes$ is natural by each argument and for each $i$ we have $F\left(p_{i}\right) \circ \otimes=\operatorname{pr}_{i}$ where $p_{i}: \prod_{j=1}^{n} X_{j} \rightarrow X_{i}$ and $\operatorname{pr}_{i}: \prod_{j=1}^{n} F X_{j} \rightarrow F X_{i}$ are natural projections.

We define the payoff functions $e u_{i}: F X_{1} \times \ldots \times F X_{n} \rightarrow \mathbb{R}$ by the formula $e u_{i}=\pi_{u_{i}} \circ \otimes$. Evidently, $e u_{i}$ is continuous. Consider any $t \in \mathbb{R}$ and $\nu \in$ $F X_{1} \times \ldots \times F X_{n}$. Then we have $\left(e u_{i}^{\nu}\right)^{-1}[t ;+\infty)=\left\{\mu_{i} \in F X_{i} \mid e u_{i}\left(\nu ; \mu_{i}\right) \geq\right.$ $\left.t_{i}\right\}=l^{-1}\left(\pi_{u_{i}}^{-1}[t ;+\infty) \cap\left\{\nu_{i}\right\} \times \ldots \times F X_{i} \times \ldots \times\left\{\nu_{n}\right\}\right)$, where $l: F X_{i} \rightarrow \prod_{j=1}^{n} F X_{j}$ 
is an embedding defined by $l\left(\mu_{i}\right)=\left(\nu ; \mu_{i}\right)$ for $\mu_{i} \in F X_{i}$. A structure of $\mathbb{F}$ algebra on the product $\prod_{j=1}^{n} F X_{j}$ of $\mathbb{F}$-algebras $\left(F X_{i}, \mu X_{i}\right)$ is given by a map $\xi: F\left(\prod_{i=1}^{n} F X_{i}\right) \rightarrow \prod_{i=1}^{n} F X_{i}$ defined by the formula $\xi=\left(\mu X_{i} \circ F\left(p_{i}\right)\right)_{i=1}^{n}$. It is easy to check that the product of convex in $F X_{i}$ sets is convex in $\prod_{i=1}^{n} F X_{i}$. Since $\mathbb{F}$ weakly preserves preimages, $\pi_{u_{i}}^{-1}[t ;+\infty)$ is convex in $\prod_{i=1}^{n} F X_{i}$. It is easy to see that $l$ is a CP map, hence the map $e u_{i}$ is quasiconcave with respect to the $i$-th variable. Hence, using Corollary 2.5, we obtain the following theorem.

TheOREM 4.1. The game with payoff functions eu $u_{i}$ has a Nash equilibrium point provided each $F X_{i}$ is connected.

\section{Games in capacities}

We need a definition of capacity on a compactum $X$. We follow the terminology of [11]. A function $c$ which assigns to each closed subset $A$ of $X$ a real number $c(A) \in[0,1]$ is called an upper-semicontinuous capacity on $X$ if the following three properties hold for any closed subsets $F$ and $G$ of $X$ :

1. $c(X)=1, c(\emptyset)=0$,

2. if $F \subset G$, then $c(F) \leq c(G)$,

3. if $c(F)<a$, then there exists an open set $O \supset F$ such that $c(B)<a$ for each compactum $B \subset O$.

We extend a capacity $c$ to all open subsets $U \subset X$ by the formula $c(U)=$ $\sup \{c(K) \mid K$ is a closed subset of $X$ such that $K \subset U\}$.

It was proved in [11] that the space $M X$ of all upper-semicontinuous capacities on a compactum $X$ is a compactum as well, if the topology on $M X$ is defined by the subbase that consists of all sets of the form $O_{-}(F, a)=$ $\{c \in M X \mid c(F)<a\}$, where $F$ is a closed subset of $X, a \in[0,1]$, and $O_{+}(U, a)=\{c \in M X \mid c(U)>a\}$, where $U$ is an open subset of $X, a \in[0,1]$. Since all capacities here are upper-semicontinuous, in the sequel we call elements of $M X$ simply capacities.

The assignment $M$ extends to the capacity functor $M$ in the category of compacta, if the map $M f: M X \rightarrow M Y$ for a continuous map of compacta $f: X \rightarrow Y$ is defined by the formula $M f(c)(F)=c\left(f^{-1}(F)\right)$, where $c \in M X$ and $F$ is a closed subset of $X$. This functor was completed to the monad $\mathbb{M}=$ $(M, \eta, \mu)$ (see [11]), where the components of the natural transformations are defined as follows: $\eta X(x)(F)=1$ if $x \in F$ and $\eta X(x)(F)=0$ if $x \notin F$; $\mu X(\mathcal{C})(F)=\sup \{t \in[0,1] \mid \mathcal{C}(\{c \in M X \mid c(F) \geq t\}) \geq t\}$, where $x \in X, F$ is a closed subset of $X$ and $\mathcal{C} \in M^{2}(X)$. 
The tensor product for capacities was considered in [7]. It is a continuous map $\otimes: M X_{1} \times \ldots \times M X_{n} \rightarrow M\left(X_{1} \times \ldots \times X_{n}\right)$. Note that, despite the space of capacities contains the space of probability measures, the tensor product of capacities in general does not extend the tensor product of probability measures.

Due to Zhou [23] we can identify the set $M X$ with some set of functionals defined on the space $C(X)$ using the Choquet integral. We consider for each $\mu \in M X$ its value on a function $f \in C(X)$ defined by the formula

$\mu(f)=\int f d \mu=\int_{0}^{\infty} \mu\{x \in X \mid f(X) \geq t\} d t+\int_{-\infty}^{0}(\mu\{x \in X \mid f(X) \geq t\}-1) d t$.

Kozhan and Zarichnyi proved in [7] the existence of Nash equilibrium for a game in capacities ef: $\prod_{i=1}^{n} M X_{i} \rightarrow \mathbb{R}^{n}$ with expected payoff functions defined by

$$
e f_{i}\left(\mu_{1}, \ldots, \mu_{n}\right)=\int_{X_{1} \times \ldots \times X_{n}} f_{i} d\left(\mu_{1} \otimes \ldots \otimes \mu_{n}\right) .
$$

Let us remark that the Choquet functional representation of capacities preserves the natural linear convexity structure on $M X$ which was used in the proof of existence of Nash equilibrium in [7]. However, this representation does not preserve the capacity monad structure.

Another functional representation of capacities was introduced in [13] (see also [10] for similar result). It uses the Sugeno integral. This representation preserves the capacity monad structure. Let us describe such a representation. Fix any increasing homeomorphism $\psi:(0,1) \rightarrow \mathbb{R}$. We put additionally $\psi(0)=$ $-\infty, \psi(1)=+\infty$ and assume that $-\infty<t<+\infty$ for each $t \in \mathbb{R}$. We consider for each $\mu \in M X$ its value on a function $f \in C(X)$ defined by the formula

$$
\mu(f)=\int_{X}^{\text {Sug }} f d \mu=\max \left\{t \in \mathbb{R} \mid \mu\left(f^{-1}([t,+\infty))\right) \geq \psi^{-1}(t)\right\} .
$$

Let us remark that we use certain modification of the Sugeno integral. The original Sugeno integral [18] "ignores" the values of a function outside the interval $[0,1]$ and we introduce a "correction" homeomorphism $\psi$ to avoid this problem. Now, following [7], we consider a game in capacities $s f: \prod_{i=1}^{n} M X_{i} \rightarrow \mathbb{R}^{n}$, but motivated by [3], we consider Sugeno expected payoff functions defined by

$$
s f_{i}\left(\mu_{1}, \ldots, \mu_{n}\right)=\int_{X_{1} \times \ldots \times X_{n}}^{\text {Sug }} f_{i} d\left(\mu_{1} \otimes \ldots \otimes \mu_{n}\right) .
$$

The question of existence of Nash equilibrium arises naturally. Since the Sugeno integral does not preserve linear convexity on $M X$, we cannot use methods from [7].

It is easy to see that $M X$ is connected for each compactum $X$. Since the capacity monad $\mathbb{M}$ is a binary $L$-monad which weakly preserves preimages with 
$\pi_{\varphi}(\nu)=\int_{X}^{\text {Sug }} f d \nu$ for any $\nu \in M X$ and $\varphi \in C(X)$ [13], we obtain the following theorem as a consequence of Theorem 4.1.

Theorem 5.1. A game in capacities sf: $\prod_{i=1}^{n} M X_{i} \rightarrow \mathbb{R}^{n}$ with Sugeno payoff functions has a Nash equilibrium point.

\section{REFERENCES}

[1] W. Briec and Ch. Horvath, Nash points, Ku Fan inequality and equilibria of abstract economies in Max-Plus and $\mathbb{B}$-convexity, J. Math. Anal. Appl. 341 (2008), 188-199.

[2] A. Chateauneuf, M. Grabisch and A. Rico, Modeling attitudes toward uncertainty through the use of the Sugeno integral, J. Math. Econom. 44 (2008), 1084-1099.

[3] D. Dubois, H. Prade and R. Sabbadin, Qualitative decision theory with Sugeno integrals, arxiv.org 1301.7372

[4] D. Dubois, J.-L. Marichal, H. Prade, M. Roubens and R. Sabbadin, The use of the discrete Sugeno integral in decision making: a survey, Internat. J. Uncertainty, Fuzziness Knowledge-Based Systems 9 (5) (2001), 539-561.

[5] I.GilboA, Expected utility with purely subjective non-additive probabilities, J. Math. Econom. 16 (1987), 65-88.

[6] D. Glycopantis And A. Muir, Nash equilibria with Knightian uncertainty; the case of capacities, Econ. Theory 37 (2008), 147-159.

[7] R. Kozhan and M. Zarichnyi, Nash equilibria for games in capacities, Econ. Theory $\mathbf{3 5}$ (2008), 321-331.

[8] S. MacLane, Categories for Working Matematicians, Springer Verlag, 1976.

[9] J. van Mill and M. VAn de Vel, Convexity preserving mappings in subbase convexity theory, Proc. Kon. Ned. Acad. Wet. 81 (1978), 76-90.

[10] O.R. Nykyforchyn, The Sugeno integral and functional representation of the monad of lattice-valued capacities, Topology 48 (2009), 137-148.

[11] O.R. NyKyforchyn AND M.M. ZaRichnyi, Capacity functor in the category of compacta, Mat. Sb. 199 (2008), 3-26.

[12] T. Radul, Convexities generated by L-monads, Appl. Categ. Structures 19 (2011), 729739 .

[13] _ A functional representation of capacity monad, Topology 48 (2009), 100-104.

[14] _ On strongly Lawson and I-Lawson monads, Bol. Mat. 6 (1999), 69-76.

[15] T.N. Radul and M.M. Zarichnyi, Monads in the category of compacta, Uspekhi Mat. Nauk. 50 (1995), 83-108.

[16] A. Rico, M. Grabisch, Ch. Labreuchea and A. Chateauneuf, Preference modeling on totally ordered sets by the Sugeno integral, Discrete Appl. Math. 147 (2005), 113-124.

[17] D. SchmeIDLER, Subjective probability and expected utility without additivity, Econometrica 57 (1989), 571-587.

[18] M. Sugeno, Fuzzy measures and fuzzy integrals, A survey, Fuzzy Automata and Decision Processes (M.M. Gupta, G.N. Saridis and B.R. Gaines, eds.), North-Holland, Amsterdam, 1977, 89-102.

[19] A.Teleiko and M. Zarichnyi, Categorical Topology of Compact Hausdorff Spaces, VNTL Publishers, Lviv, 1999

[20] M. VAn DE VeL, Theory of convex strutures, North-Holland, 1993.

[21] A.WieczoreK, The Kakutani property and the fixed point property of topological spaces with abstract convexity, J. Math. Anal. Appl. 168 (1992), 483-499. 
[22] M. ZARIChNYI, Spaces and mappings of idempotent measures, Izv. Ross. Akad. Nauk Ser. Mat. 74 (2010), 45-64.

[23] L. ZHoU, Integral representation of continuous comonotonically additive functionals, Trans. Amer. Math. Soc. 350 (1998), 1811-1822.

Manuscript received June 8, 20115 accepted December 25, 2015

\section{TARAS RADUL}

Institute of Mathematics

Casimirus the Great University

Bydgoszcz, POLAND

and

Department of Mechanics and Mathematics

Lviv National University

Lviv, UKRAINE

E-mail address: tarasradul@yahoo.co.uk 